\title{
IL DIALETTO TEDESCO DELL'ISOLA ALLOGLOTTA DI SAPPADA TESI DI LAUREA MARIA BRUNIERA
}

Das internationale Zentrum für Mehrsprachigkeit der Universität in Udine (Centro Internationale sul Plurilinguismo dell'Università degli studi di Udine) hat 2005 eine siebzig Jahre alte Diplomarbeit veröffentlicht, eine Tatsache, die eher eine Ausnahme in der Landschaft des wissenschaftlichen Publizierens darstellt, ein Wagnis, das die Aufmerksamkeit der sprachwissenschaftlichen Öffentlichkeit verdient.

Die Autorin der Diplomarbeit, Frau Maria Bruniera, hatte sich im akademischen Jahr 1937/1938 in Padova vorgenommen, den Dialekt der deutschen Sprachinsel Sappada/ Ploden, Provinz Belluno, Friaul, wissenschaftlich zu untersuchen, daher der Titel ihrer Diplomarbeit: Il dialetto tedesco dell'isola alloglotta di Sappada.

Das maschinegeschriebene Original der auf italienisch verfassten Arbeit befindet sich in der Bibliothek des oben erwähnten Zentrums, jedoch in zwei Heften, wobei das erste 437 Seiten, einschließlich acht schwarz-weißen Fotografien und acht Zeichnungen, das zweite IV + 59 Seiten enthält. Die aktuelle faximilierte Veröffentlichung erschien einbändig, mit Vorwort und Anhang beläuft sich die Seitenzahl auf 549.

Der Mentor, Prof. Carlo Tagliavini, beurteilte die Diplomarbeit höchst positiv. In der Beurteilung steht unter anderem, dass er im Jahre 1932 selber begann, das Material zur sprachlichen Bearbeitung des deutschen Dialektes in Sappada/Ploden zu sammeln, wegen anderer Verpflichtungen aber das Thema aufgab, jedoch nicht endgültig. Er schlug es nämlich der Kandidatin Maria Bruniera vor, die in dieser Gegend aufgewachsen war. Die Tatsache, dass das Thema ihre Muttersprache war, begeisterte sie. Trotzdem zog sie ihre Mutter, die lange im venezianischen Sprachgebiet lebte, nicht als Informantin heran, denn sie strebte eine möglichst objektive Beschreibung des Sappada-Dialektes, des Sappadischen an.

Die Diplomarbeit wird durch kürzere Texte zum Thema eingeführt: Ursprung der Besiedlung, Geschichte, Land und Leute. Die ethnologischen Eigenschaften der Landschaft und Leute sowie ihre materiellen und geistigen Errungenschaften sind mit schwarz-weißen Fotografien veranschaulicht, z. B. typisches Bauernhaus, Scheune, Arbeitsverfahren wie Strohschneiden, Heuernte, Faschingstyp. Unter allen Fotografien steht die phonetisch transkribierte Benennung des entsprechenden Arbeitsvorgangs oder Bauerngerätes.

Die eigentliche sprachliche Darstellung des Sappadischen setzt sich aus folgenden Kapiteln zusammen:

- Phonetik: Die Autorin benutzte die phonetische Transkribierung nach G. I. Ascoli mit einigen Modifikationen nach einem System, das, so Maria Bruniera, nicht wesentlich von der Transkribierung deutscher Dialekten abweiche.

- Morphologie: Die Morphologie des Sappadischen versteht sich hier im weitesten Sinne des linguistischen Begriffes, was den Wert der Arbeit nicht im Geringsten mindert. Die Autorin widmet sich der Beugungsparadigmen (Deklination, Komparation, Pluralbildung, Steigerung, Konjugation) der Wortarten Substantiv, Adjektiv, Verb, Pronomen, Numerale. Sie behandelt den Gebrauch vom bestimmten und unbestimmten Artikel. Bei Wortbildungs-paradigmen hebt sie die Diminutive 
hervor, indem sie behauptet: „I diminutivi sono molto usati nel sappadino (...) soprattutto nel linguaggio dei bambini. “"

- Dialektale Texte: Das Inventar der dialektalen Texte besteht aus 43 Sprichwörtern und aus 45 Texten, die verschiedenen Textsorten zuzuzählen sind: Hierher gehören z.B. Wiegenlieder, Abzählreime, Kinderspiele, Lieder, Berichte der Informanten über Heuernte, Feiertage, Sitten und Bräuche usw. Alle Texte kommen zuerst phonetisch transkribiert vor und erst dann werden sie in die „,deutsche“ Hochsprache übersetzt.

- Den lexikographischen Teil der Diplomarbeit stellt der alphabetisch angeordnete Wortschatz des Sappadischen dar. Jedes Stichwort ist mit einer etymologischen Erklärung versehen, die von italienischer und deutscher Übersetzung gefolgt wird. Der Wortschat $z$ besteht aus 3403 Einheiten, mit $\bar{a}$ beginnend $(\bar{a}=$ anche, auch) und mit 3wivlrùihbe endend ( 3 wivinùinibe = la cipolla, Zwiebel).

- Der toponomastische Teil behandelt 355 Namen der Siedlungen (insgesamt gibt es 15), Wiesen, Berge, Bächer, Wälder, Ebenen usw.

- Dem toponomastischen Teil folgen das alphabetisch angeordnete Verzeichnis der Wörterbucheinheiten ohne Anmerkungen sowie das Verzeichnis der Familiennamen und das italienische Vokabular.

Die besprochene Buchveröffentlichung der Diplomarbeit von Maria Bruniera aus dem Jahre 1937/38 wird mit der ebenso faximilierten Beurteilung von Prof. Carlo Tagliavini abgeschlossen.

Der Mut, den die Herausgeber aufbrachten, indem sie diese Diplomarbeit ,herauskramten" und in Buchform und integral publizierten, steht auch für die Tatsache, dass Manches, das längst in Vergessenheit geraten zu sein scheint, nicht an Wert verloren hat. Es kann ruhig behauptet werden, dass die Forschungsergebnisse von Maria Bruniera und Prof. Carlo Tagliavini denen einer Dissertation gleichkommen. Hut ab!

Darko Čuden 